\title{
On the spectrum of Cayley graphs
}

\section{Ghorbani and M. Songhori}

\author{
Communicated by I. Ya. Subbotin
}

\begin{abstract}
The set of eigenvalues of the adjacency matrix of a graph is called the spectrum of it. In the present paper, we introduce the spectrum of Cayley graphs of order $p q r$ in terms of character table, where $p, q, r$ are prime numbers. We also, stablish some properties of Cayley graphs of non-abelian groups with a normal symmetric connected subset.
\end{abstract}

\section{Introduction}

By investigating Cayley graphs, even more detailed information about a group can be obtained. In this paper, we study the spectral properties of Cayley graphs via the character table of underlying group.

Computing the spectrum of Cayley graphs was started by a paper of Babai [2] in 1979 and recently, this exciting research topic is received increasing attention by mathematician, see for example $[4,6,19,22]$. Ghorbani and Nowroozi in a series of articles computed the spectrum of normal and normal edge-transitive Cayley graphs of order $n$ where $n \in\left\{p^{3}, p^{2} q, p q r, 2 p q\right\}$ and $p, q, r$ are prime numbers, see [9,11-15]. But in general the spectrum of Cayley graphs of these groups is still an open problem. Here, we compute the spectrum of such Cayley graphs by means of character table. We also compute the spectrum of Cayley graphs of non-abelian groups by constructing the circulant matrices, see [9].

2010 MSC: 20D15.

Key words and phrases: Cayley graphs, symmetric set, semi-direct product, characteristic polynomial. 
In the next section, we give the necessary definitions and some preliminary results. In section three, we stablish a formula for computing the spectra of normal Cayley graphs. Finally, in section four, we compute the spectrum of Cayley graphs of order $p q r$, in general. Here, our notation is standard and mainly taken from the standard books such as [16].

\section{Definitions and preliminaries}

Here, we introduce some basic notation and terminology used throughout the paper. All graphs considered here are finite and simple. A simple graph is a graph $\Gamma$ without loops and multiple edges. The vertex set and the edge set of graph $\Gamma$ are denoted by $V(\Gamma)$ and $E(\Gamma)$, respectively. When two vertices $u$ and $v$ are endpoints of an edge, we say that they are adjacent and write $u \sim v$ to indicate this. The adjacency matrix $A$ is an $n \times n$ matrix whose $x y$-th entry is 1 if $x y \in E$ and zero otherwise.

For given graphs $\Gamma_{1}$ and $\Gamma_{2}$ their Cartesian product $\Gamma_{1} \square \Gamma_{2}$ is defined as the graph on the vertex set $V\left(\Gamma_{1}\right) \times V\left(\Gamma_{2}\right)$, where two vertices $u=$ $\left(u_{1}, u_{2}\right)$ and $v=\left(v_{1}, v_{2}\right)$ are adjacent if and only if either $\left(\left[u_{1}=v_{1}\right.\right.$ and $\left.\left.u_{2} v_{2} \in E\left(\Gamma_{2}\right)\right]\right)$ or $\left(\left[u_{2}=v_{2}\right.\right.$ and $\left.\left.u_{1} v_{1} \in E\left(\Gamma_{1}\right)\right]\right)$. It is well-known that $A\left(\Gamma_{1} \square \Gamma_{2}\right)=A\left(\Gamma_{1}\right) \otimes I+I \otimes A\left(\Gamma_{2}\right)$, where $\otimes$ denotes the Tensor (Kronecker) product see [5].

For the finite group $G$, the generating subset $S$ is symmetric if $1 \notin S$ and $S=S^{-1}$. The Cayley graph $\Gamma=\operatorname{Cay}(G, S)$ on $G$ with respect to $S$ has the vertex set $V(\Gamma)=G$ and edge set $E(\Gamma)=\{(g, s g) \mid g \in G, s \in S\}$. By this definition the Cayley graph Cay $(G, S)$ always is connected.

Theorem 1 ([1]). Let $\Gamma_{1}=\operatorname{Cay}\left(G, S_{1}\right)$ and $\Gamma_{2}=\operatorname{Cay}\left(H, S_{2}\right)$ be two Cayley graphs. Then the Cartesian product $\Gamma_{1} \square \Gamma_{2}$ is the Cayley graph of the direct product $G \times H$ with the generating subset $\left(S_{1}, 1\right) \square\left(1, S_{2}\right)$.

The characteristic polynomial $\chi(\Gamma)$ of graph $\Gamma$ with adjacency matrix $A$ is defined as $\chi(\Gamma)=\operatorname{det}(x I-A)$. It is a monic polynomial of degree $n$. The roots of the charachteristic polynomial are eigenvalues of $\Gamma$ and form the spectrum of $\Gamma$. Since all considered graphs are undirected, the adjacency matrix $A$ is symmetric. Consequently, all eigenvalues are real.

Theorem 2 ([3]). Let $A$ and $B$ is square matrices of orders $m$ and $n$, respectively. If $\lambda_{1}, \ldots, \lambda_{m}$ are eigenvalues of $A$ and $\mu_{1}, \ldots, \mu_{n}$ are the eigenvalues of $B$, then all eigenvalues of $A \otimes B$ are $\lambda_{i} \mu_{j}(\leqslant i \leqslant m, 1 \leqslant$ $j \leqslant n)$. 
It is well-known that if $A$ is a matrix of order $m$ with eigenvalues $\lambda_{i}$ and $B$ is a matrix of order $n$ with eigenvalues $\mu_{j}$, then all eigenvalues of $a A \otimes I_{n}+b I_{m} \otimes B$ are $a \lambda_{i}+b \mu_{j}$, where $1 \leqslant i \leqslant m$ and $1 \leqslant j \leqslant n$.

A circulant matrix is a square matrix generated from a vector such as $\left[a_{0}, \ldots a_{n-1}\right]$ as the first row denoted by $\left[\left[a_{0}, \ldots, a_{n-1}\right]\right]$. Successive rows use the same elements as the first row but each such row is circularly shifted by one element. All eigenvalues of this circulant matrix are $\lambda_{\omega}=$ $\sum_{i=0}^{n-1} a_{i} \omega^{i}$, where $\omega$ is an $n$-th root of unity.

Theorem $3([2])$. Let $\left(A_{i j}, 1 \leqslant i, j \leqslant l\right)$ be square matrices of order $n$ that have the complete set of eigenvectors $\left\{V_{1}, \ldots, V_{n}\right\}$ with $A_{i j} V_{k}=\alpha_{i j}^{k} V_{k}$. Let also, $B_{k}=\left[\alpha_{i j}^{k}\right]$ be square matrices of order $l$, each with a complete set of eigenvectors $\left\{U_{1}^{k}, \ldots, U_{l}^{k}\right\}$ satisfying $B_{k} U_{j}^{k}=\beta_{j}^{k} U_{j}^{k}$ for $1 \leqslant j \leqslant l$. Then a complete set of eigenvectors $\left\{W_{1}, W_{2}, \ldots, W_{n l}\right\}$ for the square matrix

$$
A=\left(\begin{array}{cccc}
A_{11} & A_{12} & \ldots & A_{1 l} \\
A_{21} & A_{22} & \ldots & A_{2 l} \\
\vdots & \vdots & \ddots & \vdots \\
A_{l 1} & A_{l 2} & \ldots & A_{l l}
\end{array}\right)
$$

is given by $W_{(k-1) l+j}=U_{j}^{k} \otimes V_{k}$, for $k=1,2, \ldots, n$ and $j=1,2, \ldots, l$. The corresponding eigenvalues are $\lambda_{(k-1) l+j}=\beta_{j}^{k}$.

In continuing of this paper, we use above results to compute the spectrum of Cayley graphs. First, we compute the spectrum of a group of order $8 n$ denoted by $V_{8 n}$ with the following presentation:

$$
V_{8 n}=\left\langle a, b, a^{2 n}=b^{4}=1, b a=a^{-1} b^{-1}, b^{-1} a=a^{-1} b\right\rangle,
$$

where $n$ is an odd number. By using the relations of this group, we conclude that this group has exactly $8 n$ elements as follows:

$$
\left\{b^{r} a^{j}, 1 \leqslant r \leqslant 4,1 \leqslant j \leqslant 2 n\right\} .
$$

It is clear that in a Cayley graph the vertex 1 correspond to identity is adjacent with all elements of $S$.

Theorem 4. The adjacency matrix of the Cayley graph $\Gamma=\operatorname{Cay}\left(V_{8 n}, S\right)$ where $S=\left\{b a^{r}, b a^{s}, b^{3} a^{k}\right\}(1 \leqslant i \leqslant 2 n-1)$ is as follows:

- $a^{i}$ is adjacent with

$$
\left\{\begin{array}{ll}
b a^{r-i}, b a^{s-i}, b^{3} a^{t-i} & 2 \mid i \\
b a^{t-i}, b^{3} a^{s-i}, b^{3} a^{r-i} & 2 \nmid i
\end{array},\right.
$$


- $b a^{i}$ is adjacent with

$$
\left\{\begin{array}{ll}
a^{t-i}, b^{2} a^{s-i}, b^{2} a^{r-i} & 2 \mid i \\
b^{2} a^{t-i}, a^{s-i}, a^{r-i} & 2 \nmid i
\end{array},\right.
$$

- $b^{2} a^{i}$ is adjacent with

$$
\left\{\begin{array}{ll}
b a^{t-i}, b^{3} a^{s-i}, b^{3} a^{r-i} & 2 \mid i \\
b a^{r-i}, b a^{s-i}, b^{3} a^{t-i} & 2 \nmid i
\end{array},\right.
$$

- $b^{3} a^{i}$ is adjacent with

$$
\left\{\begin{array}{ll}
b^{2} a^{t-i}, a^{s-i}, a^{r-i} & 2 \mid i \\
a^{t-i}, b^{2} a^{s-i}, b^{2} a^{r-i} & 2 \nmid i
\end{array},\right.
$$

Proof. The proof is straightforward.

Corollary 1. Let $G$ be a group $V_{8 n}$. The adjacency matrix of the cubic Cayley graph $\Gamma=\operatorname{Cay}(G, S)$, where $S=\left\{b a^{r}, b a^{s}, b^{3} a^{k}\right\}$ is the following circulant matrix

$$
\left(\begin{array}{cc}
0 & A \\
A^{T} & 0
\end{array}\right)
$$

where $A=[[0, B, 0, C]]=B \otimes C+E \otimes F$ and $B, C, E, F$ are the following circulant matrices:

$$
\begin{aligned}
B & =[[0, \ldots, 0, \overbrace{1}^{r}, 0, \ldots, 0, \overbrace{1}^{s}, 0, \ldots, 0]], \\
C & =[[0,1,0,0]], \\
E & =[[0, \ldots, 0, \overbrace{1}^{t}, 0, \ldots, 0]], \\
F & =[[0,0,0,1]] .
\end{aligned}
$$

Theorem 5. Suppose $\omega=e^{\frac{\pi}{n} i}$, then

1) All eigenvalues of matrix $B$ are $\lambda_{j}=\omega^{(r-1) j}+\omega^{(s-1) j}$ in which $j=0, \ldots, 2 n-1$,

2) The eigenvalues of $C$ and $F$ are $\pm i, \pm 1$,

3) The eigenvalues of $E$ are $\lambda_{j}=\omega^{j}$ where $j=0, \ldots, 2 n-1$.

One can apply Theorem 5 to compute all eigenvalues of Cayley graph $\Gamma=\operatorname{Cay}\left(V_{8 n}, S\right)$. For example, in the following example, we compute all eigenvalues on Cayley graph $\mathrm{Cay}\left(V_{24}, S\right)$. 
Example 1. Consider the tetravalent Cayley graph $\Gamma=\operatorname{Cay}\left(V_{24}, S\right)$, where $S=\left\{b a, b a^{5}, b^{3} a^{5}\right\}$. The adjacency matrix $\Gamma$ is

$$
\left(\begin{array}{cc}
0 & A \\
A^{T} & 0
\end{array}\right)
$$

where $A=[[0, B, 0, C]], B=[[1,0,0,0,1]]$ and $C=[[0,1,0,0]]$. By using Theorem 5 , all eigenvalues are:

$$
\left\{-3^{2},-\sqrt{3}^{4},-1^{6}, 1^{6}, \sqrt{3}^{4}, 3^{2}\right\} .
$$

\section{Main results and discussions}

A general linear group $G L(V)$ of vector space $V$ is the set of all $\mathcal{A} \in \operatorname{End}(V)$, where $\mathcal{A}$ is invertible. A representation of a group $G$ is a homomorphism $\alpha: G \rightarrow G L(V)$ and the degree of $\alpha$ is equal to the dimension of $V$. A trivial representation is a homomorphism $\alpha: G \rightarrow \mathbb{C}^{*}$, where $\alpha(g)=1$, for all $g \in G$. Let $\varphi: G \rightarrow G L(V)$ be a representation with $\varphi(g)=\varphi_{g}$, the character $\chi_{\varphi}: G \rightarrow \mathbb{C}$ of $\varphi$ is defined as $\chi_{\varphi}(g)=\operatorname{tr}\left(\varphi_{g}\right)$. An irreducible character is the character of an irreducible representation and the character $\chi$ is linear, if $\chi(1)=1$. We denote the set of all irreducible characters of $G$ by $\operatorname{Irr}(G)$.

A character table is a matrix whose rows and columns are correspond to the irreducible characters and the conjugacy classes of $G$, respectively.

Proposition 1 ([3]). Let $G$ and $H$ be two finite groups with character tables $\mathcal{M}(G)$ and $\mathcal{M}(H)$, respectively. Then the character table of direct product group $G \times H$ is

$$
\mathcal{M}(G \times H)=\mathcal{M}(G) \otimes \mathcal{M}(H) .
$$

The study of spectrum of Cayley graphs is closely related to irreducible characters of $G$. If $G$ is abelian, then the spectrum of $X=\operatorname{Cay}(G, S)$ can easily be determined as follows.

Theorem 6 ([21]). Let $S$ be a symmetric subset of abelian group $G$. Then the eigenvalues of adjacency matrix of $\operatorname{Cay}(G, S)$ are given by

$$
\lambda_{\varphi}=\sum_{s \in S} \varphi(s),
$$

where $\varphi \in \operatorname{Irr}(G)$. 
Let $G$ be a finite group with symmetric subset $S$. We recall that $S$ is a normal subset if and only if $S^{g}=g^{-1} S g=S$, for all $g \in G$. The following theorem is implicitly contained in [7,21].

Theorem 7. Let $G$ be a finite group with a normal symmetric subset $S$. Let $A$ be the adjacency matrix of the graph $X=\operatorname{Cay}(G, S)$. Then the eigenvalues of $A$ are given by $\left[\lambda_{\varphi}\right]^{\varphi(1)^{2}}$, where $\lambda_{\varphi}=\frac{1}{\varphi(1)} \sum_{s \in S} \varphi(s)$ and $\varphi \in \operatorname{Irr}(G)$.

Example 2. Consider the dihedral group $D_{8}$ with the following presentation

$$
D_{8}=\left\langle a, b: a^{4}=b^{2}=1, b^{-1} a b=a^{-1}\right\rangle .
$$

The character table of dihedral group $D_{8}$ is reported in Table 1. Let $S=\left\{a, a^{-1}\right\}$, then by using Theorem 7 , all eigenvalues of $D_{8}$ are:

$$
\lambda_{\chi_{1}}=\lambda_{\chi_{3}}=2, \lambda_{\chi_{2}}=\lambda_{\chi_{4}}=-2 \text { and } \lambda_{\chi_{5}}=0 .
$$

In other words, the spectrum of Cayley graph on group $D_{8}$, where $S=\left\{a, a^{-1}\right\}$ is as follows:

$$
\left\{[-2]^{2},[0],[2]^{2}\right\}
$$

\begin{tabular}{c|ccccc}
\hline $\mathcal{M}\left(D_{8}\right)$ & 1 & $g_{1}$ & $g_{2}$ & $g_{3}$ & $g_{4}$ \\
\hline$\chi_{1}$ & 1 & 1 & 1 & 1 & 1 \\
$\chi_{2}$ & 1 & -1 & 1 & 1 & -1 \\
$\chi_{3}$ & 1 & 1 & -1 & 1 & -1 \\
$\chi_{4}$ & 1 & -1 & -1 & 1 & 1 \\
$\psi_{5}$ & 2 & 0 & 0 & -2 & 0 \\
\hline
\end{tabular}

TABLE 1. The character table of $D_{8}$.

\section{Spectra of Cayley graphs of order pqr}

Given two groups $G, H$ and a group homomorphism $\varphi: H \longrightarrow A u t(G)$, the semi-direct product of $G$ and $H$ with respect to $\varphi$ denoted $G \rtimes_{\varphi} H$ (or, simply, $G \rtimes H$ ) is a new group with set $G \times H$ and multiplication operation $\left(g_{1} ; h_{1}\right)\left(g_{2} ; h_{2}\right)=\left(g_{1}^{\prime} \varphi\left(h_{1}\right) g_{2}, h_{1} h_{2}\right)$. The aim of this section is to compute the spectrum of Cayley graphs of order $p q r$ by means of semi-direct product. To do this, we first intoduce the presentation of these group. 
A Frobenius group of order $p q$ where $p$ is prime and $q \mid p-1$ is a group of order $p q$ by the following presentation:

$$
F_{p, q}=\left\langle a, b: a^{p}=b^{q}=1, b^{-1} a b=a^{u}\right\rangle,
$$

where $u$ is an element of order $q$ in multiplicative group $\mathbb{Z}_{p}^{*}$. Let $G$ be a group of order $p q r$, where $p \geqslant q>r$ are prime numbers. It is easy to see that the Sylow $p$-subgroup $P$ of $G$ is normal. This means that $G$ has the following structure:

$$
G \cong \mathbb{Z}_{p} \rtimes_{\varphi} \mathbb{Z}_{q r} \text { or } F_{q, r} .
$$

By using the concept of semi-direct product, Hölder in [17] classified all groups of order pqr. Ghorbani and Nowroozi in [10] proved that a group of order $p q r$ is isomorphic with one of the following groups:

i) $p=q=r$, in this case there are five groups of order $p^{3}$ as follows:

- $P_{1}=\mathbb{Z}_{p^{3}}$,

- $P_{2}=\mathbb{Z}_{p} \times \mathbb{Z}_{p^{2}}$,

- $P_{3}=\mathbb{Z}_{p} \times \mathbb{Z}_{p} \times \mathbb{Z}_{p}$,

- $P_{4}=\mathbb{Z}_{p} \rtimes \mathbb{Z}_{p^{2}}$,

- $P_{5}=\mathbb{Z}_{p} \rtimes\left(\mathbb{Z}_{p} \times \mathbb{Z}_{p}\right)$.

ii) $p>q>r$, then all groups of order $p q r$ are

- $G_{1}=\mathbb{Z}_{p q r}$

- $G_{2}=\mathbb{Z}_{r} \times F_{p, q}(q \mid p-1)$,

- $G_{3}=\mathbb{Z}_{q} \times F_{p, r}(r \mid p-1)$,

- $G_{4}=\mathbb{Z}_{p} \times F_{q, r}(r \mid q-1)$,

- $G_{5}=F_{p, q r}(q r \mid p-1)$,

- $G_{i+5}=\left\langle a, b, c: a^{p}=b^{q}=c^{r}=1, a b=b a, c^{-1} b c=b^{u}, c^{-1} a c=\right.$ $\left.a^{v^{i}}\right\rangle$, where $r \mid p-1, q-1, o(u)=r$ in $\mathbb{Z}_{q}^{*}$ and $o(v)=r$ in $\mathbb{Z}_{p}^{*}(1 \leqslant i \leqslant r-1)$.

iii) $p<q$ and $r=p$, then all groups of order $p^{2} q$ are

- $L_{1}=\mathbb{Z}_{p^{2} q}$,

- $L_{2}=\mathbb{Z}_{p} \times \mathbb{Z}_{p} \times \mathbb{Z}_{q}$,

- $L_{3}=\mathbb{Z}_{p} \times F_{q, p}(p \mid q-1)$,

- $L_{4}=F_{q, p^{2}}\left(p^{2} \mid q-1\right)$,

- $L_{5}=\left\langle a, b: a^{p^{2}}=b^{q}=1, a^{-1} b a=b^{\alpha}, \alpha^{p} \equiv 1(\bmod q)\right\rangle$.

iv) $q<p$ and $r=p$, then all groups of order $p^{2} q$, where $q \mid p-1$ are

- $Q_{1}=\mathbb{Z}_{p^{2} q}$

- $Q_{2}=\mathbb{Z}_{p} \times \mathbb{Z}_{q} \times \mathbb{Z}_{p}$,

- $Q_{3}=\mathbb{Z}_{p} \times F_{p, q}(q \mid p-1)$,

- $Q_{4}=F_{p^{2}, q}\left(q \mid p^{2}-1\right)$, 
- $Q_{5}=\left\langle a, b, c: a^{p}=b^{q}=c^{p}=1, a c=c a, b^{-1} a b=a^{\alpha}, b^{-1} c b=\right.$ $\left.c^{\alpha^{x}}, \alpha^{q} \equiv 1(\bmod p), x=1, \ldots, q-1\right\rangle$.

The general structures of groups $G_{i+5}, Q_{5}$ and $Q_{6}$ are in terms of semi-direct product, namely $\mathbb{Z}_{r} \ltimes\left(\mathbb{Z}_{p} \times \mathbb{Z}_{q}\right)$. In [8] the spectra of Cayley graphs Cay $\left(\mathbb{Z}_{m} \rtimes \mathbb{Z}_{n}, S\right)$ are studied. In continuing, by using some results of representation theory, we propose the spectra of Cayley graphs of order $p q r$, where $p, q$ and $r$ are prime numbers. A class of $\mathbb{Z}_{c} \ltimes\left(\mathbb{Z}_{m} \times \mathbb{Z}_{n}\right)$ has a representation as follows:

$$
\begin{aligned}
\mathbb{Z}_{c} & \ltimes_{K}\left(\mathbb{Z}_{m} \times \mathbb{Z}_{n}\right) \\
\quad & =\left\langle x, y, z ; x^{m}=y^{n}=z^{c}=1, a b=b a, z x z^{-1}=x^{k}, z y z^{-1}=y^{k^{\prime}}\right\rangle,
\end{aligned}
$$

where $o(k)=c$ in $\mathbb{Z}_{m}^{*}, o\left(k^{\prime}\right)=c$ in $\mathbb{Z}_{n}^{*}$ and $K=\left(k, k^{\prime}\right)$.

An embedding map is a homomorphism $\psi: G \longrightarrow G L_{n}(\mathbb{F})$, and we say that the representation is faithful if $\psi$ is injective. Here, we suppose that the filed $\mathbb{F}$ is complex number $\mathbb{C}$. Let $G$ is a finite group and for $g \in G, A_{g}=A(\operatorname{Cay}(G,\{g\}))$ is the adjacency matrix of related a Cayley graph. If $S \subseteq G$, then $A_{S}=A(\operatorname{Cay}(G, S))$ and according to [8, Theorem 3.2], we have $A_{S}=\sum_{s \in S} A_{s}$.

Theorem 8 ([8]). Given a group $G$ and an element $g \in G$, consider the set $\Gamma=\left\{A_{g} \mid g \in G\right\}$ and the map $\psi: G \longrightarrow \Gamma$ given by $\psi(g)=A_{g}$. Then, $\psi$ gives a faithful representation for $G$ in $G L_{|G|}(\mathbb{Q})$.

The adjacency representation of group $G$ is a representation given by $\psi$. This is called the regular representation in the literature [24].

Definition 1. Let $C_{h}$ be the $h \times h$ matrix with entries

$$
c_{i j}= \begin{cases}1, & j-i \equiv 1 \quad(\bmod h) \\ 0 & \text { otherwise. }\end{cases}
$$

This matrix is a circulant matrix or cyclic, since it is the adjacency matrix of Cayley graph $\mathbb{Z}_{h}$ with $S=\{x\}$ where $x^{h}=i d$.

Definition 2. Suppose $m, n$ and $k$ satisfies in relation $m^{k} \equiv 1(\bmod n)$. Let $\Omega_{h}$ be the $m \times m$ matrix with entries

$$
\Omega_{i j}= \begin{cases}\omega^{h k^{i-1}} & \text { when }(i=j), \\ 0 & \text { otherwise }\end{cases}
$$


where $\omega=e^{\frac{2 \pi i}{n}}$ is a primitive n-th root of unity. Clearly, for $a \in \mathbb{Z}$, $\left(\Omega_{h}\right)^{a}=\Omega_{h a}$. On the other hand, the matrix $\left(C_{h}\right)^{l}$ is given by

$$
c_{i j}= \begin{cases}1, & j-i \equiv l(\bmod h) \\ 0 & \text { otherwise. }\end{cases}
$$

Now, let $m^{k} \equiv 1(\bmod c)$ and $\left.n^{k^{\prime}} \equiv 1(\bmod c)\right)$. Suppose $X, Y$ and $Z$ are three $m n \times m n$ block matrices with the following blocks with $c \times c$ order:

$$
\begin{gathered}
X_{c \times c}=\left(\begin{array}{ccc}
\Omega_{1} & & 0 \\
& \ddots & \\
0 & & \Omega_{m n}
\end{array}\right), \quad Y_{c \times c}=\left(\begin{array}{ccc}
\Omega_{1}^{\prime} & & 0 \\
& \ddots & \\
0 & & \Omega_{m n}^{\prime}
\end{array}\right), \\
\text { and } Z_{c \times c}=\left(\begin{array}{ccc}
C_{c} & & 0 \\
& \ddots & \\
0 & & C_{c}
\end{array}\right) .
\end{gathered}
$$

It is simple to show $X^{n}=I, Y^{m}=I$ and $Z^{c}=I$. If $x, y$ and $z$ are generatores of $\mathbb{Z}_{m}, \mathbb{Z}_{n}$ and $\mathbb{Z}_{c}$, respectivley then we have:

Theorem 9. Let $G$ be a finite group and $X, Y, Z$ be block matrices of order $m n \times m n$ with blocks of order $c \times c$. Three matrices $X, Y$ and $Z$ are generators of a faithful representation of $G=\mathbb{Z}_{c} \ltimes_{K}\left(\mathbb{Z}_{m} \times \mathbb{Z}_{n}\right)$, where $K=\left(k, k^{\prime}\right)$. Then $G$ has a represention in (2), $\varphi$ is injection and $\varphi\left(x^{a} y^{b} z^{d}\right)=X^{a} Y^{b} Z^{d}$.

Proof. See [8, Theorem 3.4].

The representation $\varphi$ with conditions of Theorem 8 or 9 is called a natural representation.

Theorem 10. The natural representation and adjacency represention of $\mathbb{Z}_{c} \ltimes_{K}\left(\mathbb{Z}_{m} \times \mathbb{Z}_{n}\right)$, where $K=\left(k, k^{\prime}\right)$ are isomorphic.

Proof. We show $\operatorname{tr}\left(A_{x^{a} y^{b} z^{d}}\right)=\operatorname{tr}\left(X^{a} Y^{b} Z^{d}\right) \cdot x^{a} y^{b} z^{d}=i d$ iff $X^{a} Y^{b} Z^{d}=I$ and so $\operatorname{tr}\left(A_{i d}\right)=\operatorname{tr}(I)=m n c$. Let $x^{a} y^{b} z^{d} \neq i d$. The adjacency represention of this element is $A_{x^{a} y^{b} z^{d}}$. The Cayley graph Cay $\left(\mathbb{Z}_{c} \ltimes_{K}\left(\mathbb{Z}_{m} \times\right.\right.$ $\left.\left.\mathbb{Z}_{n}\right),\left\{x^{a} y^{b} z^{d}\right\}\right)$ is simple and so there is no a non-zero element in the main diagonal. This means that $\operatorname{tr}\left(A_{x^{a} y^{b} z^{d}}\right)=0$. The natural representation of $x^{a} y^{b} z^{d}$ is $X^{a} Y^{b} Z^{d}$, where

$$
\left(X^{a} Y^{b} Z^{d}\right)_{i j}= \begin{cases}\left(\Omega_{i}\right)^{a}\left(\Omega_{j}^{\prime}\right)^{b}\left(C_{c}\right)^{d}, & i=j, \\ 0 & \text { otherwise. }\end{cases}
$$


If $d \nmid c$, all diagonal elements in the block matrix $X^{a} Y^{b} Z^{d}$ are zero and then $\operatorname{tr}\left(A_{x^{a} y^{b} z^{d}}\right)=0$. Let $d \mid c$, if $a \nmid n$ and $b \nmid m$ then the block $\Omega_{c a} \Omega^{\prime}{ }_{c b}$ is

$$
\operatorname{tr}\left(\Omega_{c a} \Omega_{c b}^{\prime}\right)=\omega^{a} \cdot \omega^{\prime b}\left(1+\omega^{k} \cdot \omega^{\prime k^{\prime}}+\cdots+\omega^{k^{c-1}} \cdot \omega^{\prime k^{\prime c-1}}\right) .
$$

Then, for matrix $X^{a} Y^{b}$ we yield

$$
\begin{aligned}
\operatorname{tr}\left(X^{a} Y^{b}\right) & =\omega^{a} \cdot \omega^{\prime b} \alpha+\omega^{2 a} \cdot \omega^{\prime 2 b} \alpha+\cdots+\omega^{m n a} \cdot \omega^{\prime m n b} \alpha \\
& =\left(\omega^{a} \cdot \omega^{\prime b}+\omega^{2 a} \cdot \omega^{\prime 2 b}+\cdots+\omega^{m n a} \cdot \omega^{\prime m n b}\right) \alpha \\
& =\omega^{a} \cdot \omega^{\prime b} \frac{\left(\omega^{m n a} \cdot \omega^{\prime m n b}-1\right)}{\omega^{a} \cdot \omega^{\prime b}-1} \alpha,
\end{aligned}
$$

where $\alpha=\left(1+\omega^{k} \cdot \omega^{\prime k^{\prime}}+\cdots+\omega^{k^{c-1}} \cdot \omega^{\prime k^{\prime c-1}}\right)$. So $\operatorname{tr}\left(X^{a} Y^{b}\right)=0$ and these two group representations are isomorphic.

Theorem 11. The characteristic polynomial of Cayley graph Cay $(G, S)$, where $G$ is as represented in Eq. (2), is as follows:

$$
\chi\left(A\left(\mathbb{Z}_{c} \ltimes_{K}\left(\mathbb{Z}_{m} \times \mathbb{Z}_{n}\right), S\right)\right)=\prod_{i=0}^{m n-1} \chi\left(\sum_{x^{a} y^{b} z^{d}} \Omega_{i a} \Omega_{i b}^{\prime}\left(C_{c}\right)^{d}\right) .
$$

Proof. Let $G=\mathbb{Z}_{c} \ltimes_{K}\left(\mathbb{Z}_{m} \times \mathbb{Z}_{n}\right)$, where $K=\left(k, k^{\prime}\right)$ and

$$
\begin{aligned}
& \mathbb{Z}_{c} \ltimes\left(\mathbb{Z}_{m} \times \mathbb{Z}_{n}\right) \\
& \quad\left\langle x, y, z ; x^{m}=y^{n}=z^{c}=1, a b=b a, z x z^{-1}=x^{k}, z y z^{-1}=y^{k^{\prime}}\right\rangle,
\end{aligned}
$$

where $o(k)=c$ in $\mathbb{Z}_{m}^{*}$ and $o\left(k^{\prime}\right)=c$ in $\mathbb{Z}_{n}^{*}$. It yields that

$$
\chi(A(\operatorname{Cay}(G, S)))=\chi\left(\sum_{s \in S} A(\operatorname{Cay}(G,\{s\}))\right)=\chi\left(\sum_{s \in S} A_{s}\right) .
$$

The element $s \in G$ can be written uniquely as $x^{a} y^{b} z^{d}$, where $0 \leqslant a<n$, $0 \leqslant y<m$ and $0 \leqslant d<c$. Then

$$
\chi\left(\sum_{x^{a} y^{b} z^{d}} A_{x^{a} y^{b} z^{d}}\right)=\chi\left(\sum_{x^{a} y^{b} z^{d}} X^{a} Y^{b} Z^{d}\right) .
$$

Therefore

$$
\prod_{i=0}^{m n-1} \chi\left(\sum_{x^{a} y^{b} z^{d} \in S}\left(\Omega_{i}\right)^{a}\left(\Omega_{i}^{\prime}\right)^{b}\left(C_{c}\right)^{d}\right)=\prod_{i=0}^{m n-1} \chi\left(\sum_{x^{a} y^{b} z^{d} \in S}\left(\Omega_{i a}\right)\left(\Omega_{i b}^{\prime}\right)\left(C_{c d}\right)\right)
$$


By this formula, we can determine the characteristic polynomials of Cayley graphs of groups $G_{i+5}, L_{5}, P_{5}$ and $Q_{5}$.

Corollary 2. For the Frobenius group $F_{p, q}$, we have

$$
\chi\left(A\left(\mathbb{Z}_{q} \ltimes_{k} \mathbb{Z}_{p}, S\right)\right)=\prod_{i=0}^{q-1} \chi\left(\sum_{x^{a} y^{b}} \Omega_{i a}\left(C_{q}\right)^{b}\right) .
$$

This result gives characteristic polynomials of $\operatorname{Cay}(G, S)$, where $G \in$ $\left\{G_{5}, L_{4}, P_{4}, Q_{4}\right\}$. The spectrum of Cayley graphs of cyclic groups was computed in [20] and this subject is a corollary of Theorem 11.

Proposition 2 ([20]). All eigenvalues of $\mathrm{Cay}\left(\mathbb{Z}_{n} ; S\right)$ are given by

$$
\left\{\lambda \mid \lambda=\sum_{s \in S} \omega^{x s}, x \in \mathbb{Z}_{n}, 1 \leqslant x \leqslant n\right\} .
$$

Hence, the characteristic polynomials of Cayley graphs of groups $G_{1}$, $L_{1}, P_{1}$ and $Q_{1}$ are as follows:

Corollary 3. Let $x$ be a generator of cyclic group $\mathbb{Z}_{n}$ and $y$ be a generator of $\mathbb{Z}_{m}$. The eigenvalues of $\operatorname{Cay}\left(\mathbb{Z}_{n} \times \mathbb{Z}_{m}, S\right)$, where $S=\{x, y\}$ are

$$
\left\{\lambda \mid \lambda=\omega_{n}^{i}+\omega_{m}^{j}, 0 \leqslant i<n, 0 \leqslant j<m\right\} .
$$

By using Corollary 3, we can determine the spectra of Cayley graphs of groups $L_{2}, P_{2}, P_{3}$ and $Q_{2}$.

Let $\Gamma_{1}=\operatorname{Cay}\left(\mathbb{Z}_{c}, S_{1}\right)$ and $\Gamma_{2}=\operatorname{Cay}\left(\mathbb{Z}_{n} \rtimes \mathbb{Z}_{m}, S_{2}\right)$ be two Cayley graphs, then

$$
\Gamma_{1} \square \Gamma_{2} \cong \operatorname{Cay}\left(\mathbb{Z}_{c} \times\left(\mathbb{Z}_{n} \rtimes \mathbb{Z}_{m}\right), T\right),
$$

where $T=\left\{(s, 1)(1, t) \mid s \in S_{1}, t \in S_{2}\right\}$.

\section{Corollary 4.}

$$
\chi\left(\operatorname{Cay}\left(\Gamma_{1} \times \Gamma_{2}, T\right)\right)=\chi\left(\operatorname{Cay}\left(\Gamma_{1}, S_{1}\right)\right) \cdot \chi\left(\operatorname{Cay}\left(\Gamma_{2}, S_{2}\right)\right) .
$$

By using Corollary 4 one can find the characteristic polynomial of Cayley graph $\operatorname{Cay}(G, S)$, where $G \in\left\{G_{2}, G_{3}, G_{4}, L_{3}, Q_{3}\right\}$.

Example 3. Let

$$
P_{4}=\left\langle a, b: a^{p^{2}}=b^{p}=1, b^{-1} a b=a^{p+1}\right\rangle \quad \text { and } \quad S=\left\{a, a^{1}, b, b^{-1}\right\},
$$


then the adjacency matrix of $\operatorname{Cay}\left(P_{4}, S\right)$ is a $p \times p$ block matrix as following form:

$$
A=[L_{p^{2}}, I_{p^{2}}, \overbrace{0, \ldots, 0}^{p-3}, I_{p^{2}}]=L_{p^{2}} \otimes I_{p}+I_{p^{2}} \otimes[0,1,0, \ldots, 0,1],
$$

where $L_{p^{2}}=[0,1,0, \ldots, 0,1]$. Then all eigenvalues of this matrix are $2 \cos \left(\frac{2 k \pi}{p^{2}}\right)+2 \cos \left(\frac{2 k^{\prime} \pi}{p}\right)$, where $1 \leqslant k \leqslant p^{2}$ and $1 \leqslant k^{\prime} \leqslant p$.

\section{Example 4. Let}

$$
P_{5}=\left\langle a, b, c: a^{p}=b^{p}=c^{p}=1,[a, b]=c,[a, c]=[b, c]=1\right\rangle
$$

and

$$
S=\left\{a, a^{1}, b, b^{-1}\right\},
$$

then the adjacency matrix of $\operatorname{Cay}\left(P_{5}, S\right)$ is a $p^{2} \times p^{2}$ block matrix, where

$$
A=[L_{p}, I, \overbrace{0, \ldots, 0}^{p-3}, I, \overbrace{0, \ldots, 0}^{p^{2}-p}]=L_{p} \otimes I_{p^{2}}+I_{p} \otimes[0,1,0, \ldots, 0,1]_{p^{2}},
$$

and $L=[0,1,0, \ldots, 0,1]$. So all eigenvalues of this matrix are $\lambda_{k}+\mu_{k^{\prime}}$, where $1 \leqslant k \leqslant p, 1 \leqslant k^{\prime} \leqslant p^{2}$ and

$\lambda_{k}=e^{\frac{2 \pi i k}{p}}+e^{-\frac{2 \pi i k}{p}}, \quad 1 \leqslant k \leqslant p, \quad \mu_{k^{\prime}}=e^{\frac{2 \pi i k^{\prime}}{p}}+e^{-\frac{2 \pi i k^{\prime}}{p}}, \quad 1 \leqslant k^{\prime} \leqslant p^{2}$.

\section{References}

[1] A. R. Abdollahi, A. Loghman, Cayley graphs isomorphic to the product of two Cayley graphs, Ars Combin. Ars Combinatoria. 126 (2016) pp. 301-310.

[2] L. Babai, Spectra of Cayley Graphs, J. Combin. Theory Series B. 27 (1979) pp. $180-189$.

[3] R. A. Brualdi, D. Cvetković, A Combinatorial Approach to Matrix Theory and Its Applications, Chapman and Hall/CRC; Second edition, (2008).

[4] G. Chapuy and V. Féray, A note on a Cayley graph of $\mathbb{S}_{n}\langle$ ar Xiv : 1202.4976v2 .

[5] D. Cvetković, M. Doob, H. Sachs, Spectra of Graphs-Theory and Application, Deutscher Verlag der Wissenschaften, Berlin, Academic Press, third edition, Johann Ambrosius Barth Verlag, (1995).

[6] M. DeVos, L. Goddyn, B. Mohar and R. Šámal, Cayley sum graphs and eigenvalues of (3,6)-fullerenes, J. Comb. Theory Series. 99 (2009) pp. 358-369.

[7] P. Diaconis and M. Shahshahani, Generating a random permutation with random transpositions, Zeit. für Wahrsch. verw. Gebiete 57 (1981) pp. 159-179.

[8] N. Fox, Spectra of Semidirect Products of Cyclic Groups, Rose-Hulman Undergraduate Mathematics Journal 11(2) (2010) pp. 131-147. 
[9] M. Ghorbani, On the eigenvalues of normal edge-transitive Cayley graphs, Bulletin of the Iranian Mathematical Society. 1 (2014) pp. 49-56.

[10] M. Ghorbani, F. Nowroozi Larki, Automorphism group of groups of order pqr, Algebraic Structures and Their Applications. 41 (2015) pp. 101-107.

[11] M. Ghorbani, F. Nowroozi Larki, On the spectrum of Cayley graphs related to the finite groups, Filomat. 31 (2017) pp. 6419-6429.

[12] M. Ghorbani, F. Nowroozi Larki, On the spectrum of Cayley graphs, Siberian Electronic Mathematical Reports. 16 (2016) pp. 1283-1289.

[13] M. Ghorbani, F. Nowroozi Larki, On the spectrum of finite Cayley graphs, Journal of Discrete Mathematical Sciences and Cryptography, Journal of Discrete Mathematical Sciences and Cryptography. 21 (2018) pp. 83-112.

[14] M. Ghorbani, A. Seyyed-Hadi, F. Nowroozi-Larki, Computing the eigenvalues of graphs of order $p^{2} q$, Journal of Algebraic Systems. 7 (2020) pp. 189-203.

[15] M. Ghorbani, M. Songhori, M. Rajabi-Parsa, Normal edge-transitive Cayley graphs whose order are a product of three primes, Italian Journal of Pure and Applied Mathematics. 39 (2018) pp. 628-635.

[16] C. D. Godsil, G. Royle, Algebraic Graph Theory, New York, Springer, (2001).

[17] H. Hölder, Die Gruppen der Ordnungen $p^{3}, p q^{2}, p q r, p^{4}$, Math. Ann. (1893) pp. 371410.

[18] G. James, M. Liebeck, Representation and characters of groups, Cambridge University Press, Cambridge, (1993).

[19] R. Krakovski and B. Mohar, Spectrum of Cayley graphs on the symmetric group generated by transpositions, Linear Algebra Appl. 437 (2012) pp. 1033-1039.

[20] J. Lazenby, Circulant Graphs and Their Spectra, Senior Thesis, Reed College, Portland, OR, May (2008).

[21] S. L. Lee, Y. L. Luo, B. E. Sagan, Y.-N. Yeh, Eigenvectors and eigenvalues of some special graphs, IV multilevel circulants. Int. J. Quant. Chem. 41 (1992) pp.105-116.

[22] L. Lovász, Spectra of graphs with transitive groups, Period. Math. Hungar. 6 (1975) pp. 191-196.

[23] B. E. Sagan, The Symmetric Group, second ed., Springer, New York, (2001).

[24] J. Serre, Linear Representations of Finite Groups, Springer, New York, (1977).

\section{CONTACT INFORMATION}

Modjtaba Ghorbani, Department of Mathematics, Faculty of Science, Mahin Songhor Shahid Rajaee Teacher Training University, Tehran, 16785-136, I.R. Iran

$$
\begin{aligned}
E-\operatorname{Mail}(s): & \text { mghorbani@sru.ac.ir, } \\
& \text { mahinsonghori@gmail.com }
\end{aligned}
$$

Received by the editors: 03.10.2017. 